PROCEEDINGS OF THE

AMERICAN MATHEMATICAL SOCIETY

Volume 135, Number 6, June 2007, Pages 1735-1740

S 0002-9939(06)08624-2

Article electronically published on November 15, 2006

\title{
RESTRICTIONS OF BOUNDED LINEAR OPERATORS: CLOSED RANGE
}

\author{
BRUCE A. BARNES
}

(Communicated by Joseph A. Ball)

\begin{abstract}
Let $T$ be a bounded linear operator on a Banach space $X$, and let $W$ be a subspace of $X$ which is a Banach space and $T$ - invariant. Denote by $T_{W}$ the restriction of $T$ to $W$. This paper explores the questions:

- If the range of $T$ is closed, under what conditions is the range of $T_{W}$ closed?

o If the range of $T_{W}$ is closed, under what conditions is the range of $T$ closed?
\end{abstract}

\section{The BASIC SETUP}

Throughout, $X$ is a Banach space with norm $\|x\|_{X}, W$ is a subspace of $X$ such that $W$ is a Banach space with norm $\|w\|_{W}$, and $W$ is continuously embedded in $X$ (i.e., $\exists M>0$ such that $M\|w\|_{W} \geq\|w\|_{X}$ for all $w \in W$ ). We denote the algebra of all bounded linear operators defined on a normed linear space $Y$ by $B(Y)$. For $T \in B(Y)$, let $\mathbf{N}(T)$ and $\mathbf{R}(T)$ be the null space and range of $T$, respectively. When $W$ is invariant for $T \in B(X)[T(W) \subseteq W]$, we denote the restriction of $T$ to $W$ by $T_{W}$. In this case, it is straightforward to check, using the Closed Graph Theorem, that $T_{W} \in B(W)$.

We are interested in the basic questions:

When $W$ is invariant for $T \in B(X)$, (1) under what conditions is it true that $\mathbf{R}(T)$ closed in $X \Longrightarrow \mathbf{R}\left(T_{W}\right)$ is closed in $W$ ?; (2) under what conditions is it true that $\mathbf{R}\left(T_{W}\right)$ closed in $W \Longrightarrow \mathbf{R}(T)$ is closed in $X$ ? We are especially interested in conditions that give answers to either of the questions (1) or (2) for all operators of the form $\lambda-T$ and $\lambda-T_{W}, \lambda \neq 0$.

The results of this paper augment the author's paper on the spectral theory of extensions of bounded linear operators in B1].

\section{REMARKS ON THE SITUATION WHERE $W$ IS A CLOSED SUBSPACE OF $X$}

In this section we assume that $W$ is always a closed subspace of $X$. We start with a simple example. Let $T \in B(X)$ have the properties that $\mathbf{N}(T) \neq\{0\}$ and $\mathbf{R}(T)$ is not closed in $X$. Setting $W=\mathbf{N}(T)$, we have $\mathbf{R}\left(T_{W}\right)$ is closed, but by assumption, $\mathbf{R}(T)$ is not closed.

Received by the editors September 9, 2005 and, in revised form, December 28, 2005.

2000 Mathematics Subject Classification. Primary 47A05.

Key words and phrases. Restriction of a bounded linear operator, closed range.

(C) 2006 American Mathematical Society 
Example 1. Assume that $T \in B(X)$ has the properties that $\mathbf{R}(T)$ is closed in $X$, but $\mathbf{R}\left(T^{2}\right)$ is not closed in $X$ (we outline how to construct such an operator below). Set $W=\mathbf{R}(T)$. Then $\mathbf{R}\left(T_{W}\right)=\mathbf{R}\left(T^{2}\right)$ is not closed in $W$.

Let $H$ be a separable Hilbert space with closed subspaces $J$ and $K$ such that $J+K$ is not closed; there is an example given in $[\mathrm{H}$, section 15]. Let $P$ and $Q$ be the orthogonal projections with $\mathbf{R}(P)=K$ and $\mathbf{R}(Q)=J^{\perp}$. Since $K$ and $J^{\perp}$ are both separable Hilbert spaces which are not finite dimensional, there exists a linear isometry $V$ mapping $J^{\perp}$ onto $K$. Set $T=P V Q \in B(H)$. Now if $T x=0$, then $P(V Q x)=0 \Longrightarrow V Q x \in K^{\perp} \Longrightarrow V Q x=0 \Longrightarrow Q x=0$ (since $V$ is an isometry on $J^{\perp}$ and $\left.Q x \in J^{\perp}\right)$. Therefore, $x \in J$. This shows that $\mathbf{N}(T)=J$. Clearly, $\mathbf{R}(T)=K$. Thus, $T$ has closed range and $\mathbf{R}(T)+\mathbf{N}(T)=K+J$, which is not closed.

Claim. $\mathbf{R}\left(T^{2}\right)$ is not closed.

To verify the Claim, first note that $T(\mathbf{R}(T)+\mathbf{N}(T))=\mathbf{R}\left(T^{2}\right)$. Also, if $T x \in$ $\mathbf{R}\left(T^{2}\right)$, then $T x=T^{2} y$ for some $y$. Therefore $x-T y \in \mathbf{N}(T)$, so $x=T y+(x-T y)$ $\in \mathbf{R}(T)+\mathbf{N}(T)$. This shows that the inverse image $T^{-1}\left[\mathbf{R}\left(T^{2}\right)\right]=\mathbf{R}(T)+\mathbf{N}(T)$. Since $\mathbf{R}(T)+\mathbf{N}(T)$ is not closed, it follows that $\mathbf{R}\left(T^{2}\right)$ is not closed.

We note the basic criterion: $T \in B(X)$ has closed range in $X$ if and only if $\exists \gamma>0$ such that $\|T(x)\|_{X} \geq \gamma\|x+\mathbf{N}(T)\|_{q}$ for all $x \in X$ (here $\|x+\mathbf{N}(T)\|_{q}$ is the usual quotient norm on $X / \mathbf{N}(T))$. Also, we use the standard terminology: $T \in$ $B(X)$ is bounded below if $\exists \gamma>0$ such that $\|T(x)\|_{X} \geq \gamma\|x\|_{X}$ for all $x \in X$. As is well known, $T$ is bounded below exactly when $\mathbf{N}(T)=\{0\}$ and $\mathbf{R}(T)$ is closed. Clearly, when $T \in B(X)$ is bounded below and $W$ is a closed $T$-invariant subspace of $X$, then $T_{W}$ is bounded below.

\section{The MAIN RESUlts}

In this section we give some answers to the questions stated in the introductory section, section 1 .

Proposition 2. Assume that $S \in B(X)$ and $W$ is $S$-invariant. The statements (a) and (b) are equivalent:

(a) $S^{-1}[W] \equiv\{x \in X: S(x) \in W\}=W+\mathbf{N}(S)$;

(b) $\mathbf{R}\left(S_{W}\right)=\mathbf{R}(S) \cap W$.

When (b) holds, $\mathbf{R}(S)$ closed $\Longrightarrow \mathbf{R}\left(S_{W}\right)$ is closed.

Proof. Suppose that (a) holds. Now always $\mathbf{R}\left(S_{W}\right) \subseteq \mathbf{R}(S) \cap W$. We prove the reverse inclusion. Suppose $y \in X$ and $S(y)=w \in W$. Then by (a), $y=v+z$ where $v \in W$ and $z \in \mathbf{N}(S)$. Then $w=S(v)$, so $w \in \mathbf{R}\left(S_{W}\right)$.

Now suppose that (b) is true. If $y \in X$ and $S(y)=w \in W$, then there exists $v \in W$ such that $S(v)=S(y)$. Then $y=v+(y-v)$ and $(y-v) \in \mathbf{N}(S)$. Thus, (a) holds.

Assume that (b) holds and that $\mathbf{R}(S)$ is closed. Suppose that $\left\{w_{n}\right\} \subseteq W, w_{0} \in W$, and $\left\|S\left(w_{n}\right)-w_{0}\right\|_{W} \rightarrow 0$. By our standing hypothesis, $\left\|S\left(w_{n}\right)-w_{0}\right\|_{X} \rightarrow 0$. Then $w_{0} \in \mathbf{R}(S) \cap W$. By (b), $w_{0}=S(v)$ for some $v \in W$. This proves that $\mathbf{R}\left(S_{W}\right)$ is closed.

Let $P=\left\{S \in B(X): S(W) \subseteq W\right.$ and for some $\left.n \geq 1, S^{n}(X) \subseteq W\right\}$.

Proposition 3. Assume that $T \in P$, and fix $n, n \geq 1$, such that $T^{n}(X) \subseteq W$. Then for all $\lambda \neq 0,(\lambda-T)^{-1}[W]=W$. It follows that 
(\#) for all $\lambda \neq 0, \mathbf{R}(\lambda-T)$ closed $\Longrightarrow \mathbf{R}\left(\lambda-T_{W}\right)$ is closed.

Proof. Fix $\lambda \neq 0$. Set $S=\lambda^{-1} T$, so $S^{n}(X) \subseteq W$. Suppose $x \in X$ and $(I-S) x=$ $w \in W$. Now $I-S^{n}=(I-S) p(S)$, where $p(S)=\left(I+S+\ldots+S^{n-1}\right)$. Therefore, $\left(I-S^{n}\right) x=p(S)(I-S) x=p(S) w \in W$. Since $S^{n} x \in W$, we have $x \in W$. This proves $(\lambda-T)^{-1}[W]=(I-S)^{-1}[W]=W$.

Then the assertion (\#) follows from Proposition 2.

Note that Example 1 shows that Proposition 3 need not hold for the case where $\lambda=0$.

Proposition 4. Assume that $S \in B(X), W$ is $S$-invariant, and $S(X) \subseteq W$. If $\lambda \neq 0$ and $Z \equiv \mathbf{R}\left(\lambda-S_{W}\right)$ is closed in $W$, then $\mathbf{R}(\lambda-S)$ is closed.

Proof. First note that $S \in B(X, W)$ (this follows from the Closed Graph Theorem and the easily established fact that $S: X \rightarrow W$ is closed). We may assume that $\lambda=1$, so our hypothesis is that $Z \equiv \mathbf{R}\left(I-S_{W}\right)$ is closed in $W$. For convenience, set $\mathbf{N}=\mathbf{N}\left(I-S_{W}\right)$, and also note that $\mathbf{N}=\mathbf{N}(I-S)$ (this follows from $S(X)$ $\subseteq W)$. Define $\left(\widehat{I-S_{W}}\right): W / \mathbf{N} \rightarrow Z$ by $\left(\widehat{I-S_{W}}\right)(w+\mathbf{N})=\left(I-S_{W}\right) w$. Let $L:$ $Z \rightarrow W / \mathbf{N}$ be the bounded inverse of $\left(\widehat{I-S_{W}}\right)$ (the Open Mapping Theorem), so

$$
L\left(\widehat{I-S_{W}}\right)(w+\mathbf{N})=w+\mathbf{N} \text { for all } w \in W .
$$

Now we complete the proof that $\mathbf{R}(I-S)$ is closed by showing that the linear map $(I-S) x \rightarrow x+\mathbf{N}$ from $\mathbf{R}(I-S)$ onto $X / \mathbf{N}$ is continuous. Assume that $\left\{x_{n}\right\} \subseteq X$ and $\left\|x_{n}-S\left(x_{n}\right)\right\|_{X} \rightarrow 0$. Then $\left\|\left(I-S_{W}\right)\left(S\left(x_{n}\right)\right)\right\|_{W}=\left\|S\left(x_{n}-S\left(x_{n}\right)\right)\right\|_{W} \rightarrow 0$. Thus, $S\left(x_{n}\right)+\mathbf{N}=L\left(\widehat{I-S_{W}}\right)\left(S\left(x_{n}\right)+\mathbf{N}\right) \rightarrow 0$ in $W / \mathbf{N}$, and therefore also in $X / \mathbf{N}$. It follows that

$$
x_{n}+\mathbf{N}=\left[x_{n}-S\left(x_{n}\right)+\mathbf{N}\right]+\left[S\left(x_{n}\right)+\mathbf{N}\right] \rightarrow 0 \text { in } X / \mathbf{N} .
$$

We note that [B2, Theorem 5] could be used to prove Proposition 4. Here we have chosen to give a direct self-contained argument.

Lemma 5. Assume that $T \in B(X)$ and $T(W) \subseteq W$. Set $V=T^{-1}[W]$, so $W \subseteq$ $V \subseteq X$. Define a norm on $V$ by $\|v\|_{V}=\|v\|_{X}+\|T v\|_{W}$.

(a) $\|v\|_{V}$ is a complete norm on $V$;

(b) $W$ is continuously embedded in $V$, and $V$ is continuously embedded in $X$;

(c) $V$ is T-invariant and $T(V) \subseteq W$.

Proof. The proofs of the assertions (a), (b), and (c) are all straightforward. We provide the proof of (a).

Assume that $\left\{v_{n}\right\} \subseteq V$ is a Cauchy sequence in the norm $\|\cdot\|_{V}$. Then $\left\{v_{n}\right\}$ is Cauchy in $\|\cdot\|_{X}$ and $\left\{T\left(v_{n}\right)\right\}$ is Cauchy in $\|\cdot\|_{W}$. There exist $v_{0} \in X$ and $w_{0} \in W$ such that

$$
\left\|v_{n}-v_{0}\right\|_{X} \rightarrow 0 \text { and }\left\|T\left(v_{n}\right)-w_{0}\right\|_{W} \rightarrow 0 .
$$

Therefore, $\left\|T\left(v_{n}\right)-T\left(v_{0}\right)\right\|_{X} \rightarrow 0$ and $\left\|T\left(v_{n}\right)-w_{0}\right\|_{X} \rightarrow 0$. Thus, $T\left(v_{0}\right)=w_{0}$. This proves that $\left\|v_{n}-v_{0}\right\|_{V}=\left\|v_{n}-v_{0}\right\|_{X}+\left\|T\left(v_{n}-v_{0}\right)\right\|_{W} \rightarrow 0$.

Theorem 6. Assume that $T \in P$, and fix $n, n \geq 1$, such that $T^{n}(X) \subseteq W$. For all $\lambda \neq 0$ :

(1) $\mathbf{R}(\lambda-T)$ closed $\Longleftrightarrow \mathbf{R}\left(\lambda-T_{W}\right)$ is closed;

(2) $\mathbf{N}(\lambda-T)=\mathbf{N}\left(\lambda-T_{W}\right)$;

(3) $\lambda-T$ is bounded below $\Longleftrightarrow \lambda-T_{W}$ is bounded below. 
Proof. For convenience, set $V_{0}=W, V_{n}=X$, and $\|w\|_{0}=\|w\|_{W}$ for $w \in W$.

Let $V_{1}=T^{-1}\left[V_{0}\right]$ be equipped with the complete norm $\|v\|_{1}=\|v\|_{X}+\|T v\|_{0}$. By Lemma $5, V_{0} \subseteq V_{1} \subseteq X$, the embeddings are continuous, and $T\left(V_{1}\right) \subseteq V_{0}$. Continuing inductively in this fashion, using Lemma 5 , construct $V_{k}, 1 \leq k \leq n-1$, with norm $\|v\|_{k}=\|v\|_{X}+\|T v\|_{k-1}$, having the properties:

(a) $\left(V_{k},\|v\|_{k}\right)$ is a Banach space, $1 \leq k \leq n-1$;

(b) $W=V_{0} \subseteq V_{1} \subseteq V_{2} \subseteq \ldots \subseteq V_{n-1} \subseteq V_{n}=X$, and each of the embeddings are continuous;

(c) $T\left(V_{k}\right) \subseteq V_{k-1}$ for $1 \leq k \leq n$.

Let $T_{k}$ be the restriction of $T$ to $V_{k}, 0 \leq k \leq n$. It follows from Propositions 3 and 4 that for all $\lambda \neq 0$ and $1 \leq k \leq n, \mathbf{R}\left(\lambda-T_{k-1}\right)$ closed $\Longleftrightarrow \mathbf{R}\left(\lambda-T_{k}\right)$ is closed. Therefore, $\mathbf{R}(\lambda-T)$ closed $\Longleftrightarrow \mathbf{R}\left(\lambda-T_{W}\right)$ is closed. This proves (1).

To verify (2), first note that $\mathbf{N}\left(\lambda-T_{W}\right) \subseteq \mathbf{N}(\lambda-T)$. Now suppose that $\lambda \neq 0$ and $x \in \mathbf{N}(\lambda-T)$. Then $T x=\lambda x, T^{n} x=\lambda^{n} x$, and since $T^{n}(X) \subseteq W, x \in W$. Therefore, $\mathbf{N}(\lambda-T)=\mathbf{N}\left(\lambda-T_{W}\right)$.

(3) follows from (1) and (2).

For $S \in B(X)$, let $\sigma_{a p}(S)=\left\{\lambda: \exists\left\{x_{n}\right\} \subseteq X\right.$ with $\left\|x_{n}\right\|_{X}=1$ for all $n$, and $\left.\left\|(\lambda-S) x_{n}\right\|_{X} \rightarrow 0\right\}$. It is well known that $\sigma_{a p}(S)=\{\lambda: \lambda-S$ is not bounded below $\}$; see [L:T, p. 282]. We set $\sigma_{a p}^{\prime}(S)=\sigma_{a p}(S) \backslash\{0\}$ (set difference).

Corollary 7. Assume that $T \in P$. Then $\sigma_{a p}^{\prime}(T)=\sigma_{a p}^{\prime}\left(T_{W}\right)$.

We note that for $T \in P$, many of the results in B1 apply to $T$. This follows by using the results in B1 in combination with the construction of the spaces $V_{k}$ with the properties (a), (b), and (c) as in the proof of Theorem 6. For example: Let $\Phi(X)$ denote the set of all Fredholm operators in $B(X)$. Using [B1, Theorem $4]$ and the construction metioned above we have for all $\lambda \neq 0$,

$$
\lambda-T \in \Phi(X) \Longleftrightarrow \lambda-T_{W} \in \Phi(W) .
$$

\section{Generalized inverses}

An operator $S \in B(X)$ has a g-inverse (generalized inverse) $G \in B(X)$ if $S G S=$ $S$. There is a well-known characterization of when $S$ has a g-inverse $\mathrm{L}: \mathrm{T}$, Theorem 12.9 , p. 251]:

$S \in B(X)$ has a g-inverse $\Longleftrightarrow \mathbf{R}(S)$ is closed and has a closed complement in $X$, and $\mathbf{N}(S)$ has a closed complement.

In particular, when $X$ is a Hilbert space, $S \in B(X)$ has a g-inverse exactly when $\mathbf{R}(S)$ is closed.

Theorem 8. Assume that $T \in P$. For $\lambda \neq 0$, if $\lambda-T$ has a g-inverse in $B(X)$, then $\lambda-T_{W}$ has a g-inverse in $B(W)$. Conversely, if $W$ is dense in $X$, and $\lambda-T_{W}$ has a g-inverse in $B(W)$, then $\lambda-T$ has a g-inverse in $B(X)$.

Proof. First we verify that it suffices to prove the result when $\lambda=1$. Note that for $\lambda \neq 0, \lambda^{-1} T \in P$, and that $\left(I-\lambda^{-1} T\right)$ has a g-inverse $G$, so

$$
\left(I-\lambda^{-1} T\right) G\left(I-\lambda^{-1} T\right)=\left(I-\lambda^{-1} T\right),
$$

if and only if $(\lambda-T)\left(\lambda^{-1} G\right)(\lambda-T)=(\lambda-T)$.

Now fix $m, m \geq 1$, such that $T^{m}(X) \subseteq W$. Assume that $I-T$ has a g-inverse $G \in B(X)$. By [B3, Prop. 2], there exists a polynomial $p(x)$ with $p(0)=0$ such that $F=I+p(T)+T^{m} G T^{m}$ is a g-inverse of $I-T$. Clearly, $F(W) \subseteq W$ (since 
$\left.T^{m}(X) \subseteq W\right)$. Conversely, assume that $W$ is dense in $X$, and $I-T_{W}$ has a ginverse $G \in B(W)$. Then as noted above, $F=I+p\left(T_{W}\right)+T_{W}^{m} G T_{W}^{m} \in B(W)$ is a g-inverse of $I-T_{W}$. Now $T^{m} \in B(X, W)$, so $T^{m} G T^{m} \in B(X, W)$. Then as $W$ is continuously embedded in $X, F$ has an extension $\bar{F}=I+p(T)+T^{m} G T^{m} \in B(X)$. Note that since $W$ is dense in $X$ and $\left(I-T_{W}\right) F\left(I-T_{W}\right)=\left(I-T_{W}\right)$ on $W$, then $(I-T) \bar{F}(I-T)=(I-T)$ on $X$.

Corollary 9. Assume that $T \in P$, and $\lambda \neq 0$. Consider the following four properties:

(1) $\mathbf{R}(\lambda-T)$ is closed;

(2) $\lambda-T$ has a g-inverse in $B(X)$;

(3) $\lambda-T_{W}$ has a g-inverse in $B(W)$;

(4) $\mathbf{R}\left(\lambda-T_{W}\right)$ is closed.

If $X$ is a Hilbert space, or $W$ is dense in $X$ and $W$ is a Hilbert space, then all four conditions are equivalent.

\section{Some EXAmples}

In this section we present some samples of standard types of integral operators to which the results of section 3 apply.

Example 10. When $\Omega$ is a locally compact Hausdorff space, let $\mathrm{BC}(\Omega)$ denote the space of all functions $f: \Omega \rightarrow \mathbf{C}$ such that $f$ is bounded and continuous on $\Omega$, equipped with the complete norm, $\|f\|_{u}=\sup \{|f(\omega)|: \omega \in \Omega\}$. Let $\mu$ be a regular Borel measure on $\Omega$. The Banach space $L^{1}(\Omega, \mu) \cap B C(\Omega)$ is an important space in the theory of integral operators that act on spaces of continuous functions; see [J], 10.1 and 12.4].

Now let $G$ be a locally compact Hausdorff topological group. We assume that $G$ is unimodular and has a fixed Haar measure. For $f \in L^{1}(G)$, define $T_{f}$ on any $g \in L^{p}(G)$ by $T_{f}(g)=f * g$ (convolution). Using the convolution results summarized in [H:R, pp. 297-298], we have:

(1) If $f \in L^{1}(G) \cap L^{2}(G)$, then $T_{f}\left(L^{2}(G)\right) \subseteq L^{2}(G) \cap B C(G)$; and

(2) If $f \in L^{1}(G) \cap L^{2}(G)$, then $f * f \in L^{1}(G) \cap B C(G)$, and $T_{f}^{2}=T_{f * f}$ so $T_{f}^{2}\left(L^{1}(G)\right) \subseteq L^{1}(G) \cap B C(G)$.

Thus, the results of this paper apply to the convolution operators $T_{f}$ in these two cases. For example, in (2) we can take $X=L^{1}(G)$ and $W=L^{1}(G) \cap B C(G)$. For $f \in L^{1}(G) \cap L^{2}(G)$, we have $T_{f}^{2}(X) \subseteq W$.

Example 11. For $n \geq 1, B C^{n}(0, \infty)$ is the set of all functions $f:(0, \infty) \rightarrow \mathbf{C}$ such that for $0 \leq k \leq n, f^{(k)}$ (the $\mathrm{k}^{t h}$ derivative of $f ; f^{(0)}=f$ ) exists and $f^{(k)} \in$ $B C(0, \infty)$. The natural complete norm on $B C^{n}(0, \infty)$ is $\|f\|=\sum_{k=0}^{n}\left\|f^{(k)}\right\|_{u}$.

Fix $n \geq 1$, and assume that $g \in B C^{n-1}(0, \infty) \cap L^{1}(0, \infty), k \in B C^{n}(0, \infty)$. Set

$$
T(f)(x)=k(x) \int_{0}^{x} g(t) f(t) d t, \quad f \in B C(0, \infty), x>0 .
$$

If $f \in B C^{m}(0, \infty)$ for some $m, 0 \leq m<n$ (here $B C^{0}(0, \infty)=B C(0, \infty)$ ), then it is easily verified that $T(f) \in B C^{m+1}(0, \infty)$. Set $W=B C^{n}(0, \infty)$. We have $T^{n}(B C(0, \infty)) \subseteq W$, so the results of this paper apply to the operator $T$.

The same statements apply to much more general Volterra-type operators on $B C(0, \infty)$. 


\section{REFERENCES}

[B1] B. Barnes, The spectral and Fredholm theory of extensions of bounded linear operators, Proc. Amer. Math. Soc. 105 (1989), 941-949. MR0955454 (89i:47008)

[B2] B. Barnes, Common operator properties of the linear operators RS and SR, Proc. Amer. Math. Soc. 126 (1998), 1055-1061. MR1443814 (98f:47003)

[B3] B. Barnes, Generalized inverses of operators in some subalgebras of B(X), Acta Sci. Math.. (Szeged) 69 (2003), 349-357. MR.1991672 (2004c:47001)

[H] P. Halmos, Introduction to Hilbert space, 2nd Edition, Chelsea Pub. Co., New York, 1957. MR 1653399 (99g:47001)

[H:R] E. Hewitt and K. Ross, Abstract Harmonic Analysis, Vol. I, Springer-Verlag, Berlin, 1963. MR.0156915 (28:158)

[J] K. Jorgens, Linear Integral Operators, Pitman, Boston, London, Melbourne, 1982. MR0647629 (83j:45001)

[L:T] D. Lay and A. Taylor, Introduction to Functional Analysis, 2nd Ed., Wiley, New York, 1980. MR0564653 (81b:46001)

Department of Mathematics, University of Oregon, Eugene, Oregon 97403

E-mail address: barnes@uoregon.edu 